\title{
Presence of Comorbidities Associated with Severe Coronavirus Infection in Patients with Inflammatory Bowel Disease
}

\author{
Rajen Parekh', ${ }^{1,0} \cdot$ Xian Zhang $^{1} \cdot$ Ryan C. Ungaro ${ }^{2} \cdot$ Erica J. Brenner $^{1} \cdot$ Manasi Agrawal $^{2} \cdot$ Jean-Frederic Colombel $^{2}$. \\ Michael D. Kappelman ${ }^{1}$
}

Received: 23 March 2021 / Accepted: 13 May 2021 / Published online: 28 June 2021

(c) The Author(s) 2021

\begin{abstract}
Background Comorbidities increase the risk of coronavirus disease 2019 (COVID-19) hospitalization and mortality. As many comorbidities are common in patients with inflammatory bowel diseases (IBD), we sought to investigate the effects of comorbidities in these patients on infection severity.

Aim To evaluate association between individual comorbidities and COVID-19 infection severity among patients with IBD. Methods Data were obtained from SECURE-IBD, an international registry created to evaluate COVID-19 outcomes in patients with IBD. We used multivariable regression to analyze associations between eleven non-IBD comorbidities and a composite primary outcome of COVID-19-related hospitalization or death. Comorbidities were first modeled individually, adjusting for potential confounders. Next, to determine the independent effect of comorbidities, we fit a model including all comorbidities as covariates.

Results We analyzed 2,035 patients from 58 countries (mean age 42.7 years, 50.6\% male). A total of 538 patients (26.4\%) experienced severe COVID-19. All comorbidities but a history of stroke and obesity were associated with severe infection in our initial analysis, with adjusted odds ratios ranging from 1.9 to 3.7. In a model including all comorbidities significantly associated with the composite outcome in the initial analysis, as well as other confounders, most comorbidities remained significant, with the highest risk in chronic kidney disease and chronic obstructive pulmonary disease.

Conclusion Many non-IBD comorbidities are associated with a two to threefold increased risk of COVID-19 hospitalization or death among patients with IBD. These data can be used to risk-stratify and guide treatment and lifestyle decisions during the ongoing pandemic.
\end{abstract}

Keywords Inflammatory bowel disease $\cdot$ Crohn's disease $\cdot$ Ulcerative colitis $\cdot$ Coronavirus disease $\cdot$ Chronic kidney disease $\cdot$ Chronic obstructive pulmonary disease

\section{Abbreviations}

5-ASA

aOR

5-Aminosalicylates

Adjusted odds ratio

An editorial commenting on this article is available at https://doi. org/10.1007/s10620-021-07106-y.

Rajen Parekh

rajennparekh@gmail.com

1 Division of Pediatric Gastroenterology, Department of Pediatrics, University of North Carolina at Chapel Hill, Chapel Hill, NC, USA

2 The Henry D. Janowitz Division of Gastroenterology, Icahn School of Medicine at Mount Sinai, New York, NY, USA

3 Paul D. Schreiber High School, 101 Campus Drive, Port Washington, NY 11050, USA
CD Crohn's disease

CI Confidence interval

CKD Chronic kidney disease

COPD Chronic obstructive pulmonary disease

COVID-19 Coronavirus disease 2019

CRP C-reactive protein

IBD Inflammatory bowel disease

SARS-CoV-2 Severe acute respiratory syndrome coronavirus 2

SECURE-IBD Surveillance Epidemiology of Coronavirus Under Research Exclusion for Inflammatory Bowel Disease 


\section{Introduction}

Coronavirus disease 2019 (COVID-19) is a respiratory illness caused by the severe acute respiratory syndrome coronavirus 2 (SARS-CoV-2) virus. Although COVID-19 infection generally presents as a mild or moderate illness, the infection can cause hospitalization, need for respiratory support, and death [1,2]. Since its emergence in December 2019, COVID-19 has spread to over 200 different countries, resulting in over 123 million infections and 2.7 million deaths as of mid-March 2021[3]. Worldwide estimates for mortality due to COVID-19 are estimated to range from $0.01 \%$ to over $20 \%$, depending on patient age $[4,5]$. The severity of disease is also impacted by factors such as age, sex, and comorbidities [6]. In particular, the presence of hypertension, diabetes, chronic obstructive pulmonary disease (COPD), and cancer are important predictors of severe COVID-19 infection [7].

The effect of inflammatory bowel disease (IBD) and its treatments on the severity of COVID-19 infections is less understood. Two previous multicenter studies evaluated the risk of COVID-19 infection in patients with IBD, both finding the disease may present more severely in these patients when compared to the general population. These studies separately found hospitalization rates of patients with IBD who contracted COVID-19 to be close to $30 \%$, much greater than seen in the general population $[8,9]$. Patients with IBD may be predisposed to these more severe clinical outcomes compared to the larger, unaffected population for a number of reasons. First, immune-mediated diseases such as IBD can be associated with ongoing systemic inflammation and may predispose to thrombosis [10, 11]. Both elevated C-reactive protein (CRP) and d-Dimer levels are predictors of the severity of COVID-19 infection, and as patients with IBD may start from an initial position of vulnerability, it is possible that COVID-19 outcomes are worse in these patients [12,13]. Second, the frequent use of immunosuppressant medications may also affect the clinical course of COVID-19 infection. It has previously been demonstrated that patients with IBD on immunosuppressant medication are at greater risk for morbidity from certain infectious processes, and this may be the case for COVID-19 as well [14]. Conversely, corticosteroid treatment and possibly other immunosuppressant therapy may provide a mortality benefit in severe COVID19 infection, likely by blunting the effects of the cytokine mediated surge [14-16]. Finally, patients with IBD have a significantly higher prevalence of comorbid medical conditions, including respiratory disease, cardiovascular disease, and cancer compared to patients without IBD [17]. The presence of these comorbidities may place patients with IBD at risk for more severe COVID-19 infection, and indeed, existing multicenter research mentioned previously has demonstrated that a higher Charlson Comorbidity Index is associated with more severe COVID-19 outcomes [8].

Our group has previously published research utilizing an international database of patients with IBD who were infected with COVID. A key finding was that, in patients with IBD, increasing age and number of comorbidities were significantly associated with the severity of COVID19 [18]. However, the relative effect of specific comorbidities in patients with IBD has not yet been established. We aimed to evaluate the impact of individual comorbidities on COVID-19 severity among patients with IBD. Based on prior research demonstrating associations between comorbidities and severe COVID-19 outcomes in the general population, as well as in patients with IBD, we hypothesized that most, if not all, comorbidities would be significantly associated with greater COVID-19 severity [6-8].

\section{Methods}

\section{Data Source}

This research utilized the Surveillance Epidemiology of Coronavirus Under Research Exclusion for Inflammatory Bowel Disease (SECURE-IBD) database. The creation of this database has been previously described [18]. In brief, healthcare providers reported demographic information of patients with IBD with confirmed COVID-19, as well as IBD treatments and outcomes of COVID-19 infection. Furthermore, reporters provided information regarding eleven comorbidities: asthma, cancer, cardiovascular disease, chronic liver disease, CKD, diabetes, a history of stroke, hypertension, obesity, COPD, and other chronic lung disease. Reporters were asked "Does the patient have any of the following comorbidities (check all that apply)?" and provided with a list of comorbidities to select from, as well as the opportunity to enter any other comorbidities afflicting the patient. Reporters are requested to submit cases after one week of symptoms and sufficient time has passed to observe the clinical course of the illness through resolution or death [18].

\section{Quality Control}

We identified potential duplicate records with matching age, sex, IBD disease type, country, and state (United States only), and reviewed these manually. Reports from nonvalid email addresses were flagged as potential errors and we performed a Google search of reporters and practice locations to confirm legitimacy of reports. 


\section{Statistical Analyses}

This analysis utilized all reports to SECURE-IBD through November 8, 2020. Patient and disease characteristics were summarized by means and standard deviations for the continuous variables and by count and percentages for the categorical variables (Table 1). Our primary outcome was COVID-19 related hospitalization or death. We first analyzed associations between each individual comorbidity and COVID-19 hospitalization or death using separate logistic regressions. Covariates included patient age and sex, as well as other variables that were significantly different between patients with and without the comorbidity in bivariate analysis.

We next examined the independent effect of each comorbidity, adjusting for other comorbidities as well as patient age, patient sex, and IBD medication use, including 5-aminosalicylates (ASA), biologic therapy (any tumor necrosis factor, IL-12/23, or integrin antagonist), and corticosteroids using logistic regression. Comorbidities found to not be statistically significant using separate logistic regression models were not incorporated into the final multivariable model.

All statistical analyses were carried out in R (R Core Team, 2019) and used the Tidyverse package $[19,20]$. An alpha level $<0.05$ was considered statistically significant for all comparisons.

\section{Results}

Data from 58 countries describing 2035 COVID-19 cases in patients with IBD were reported to the SECURE-IBD registry at the time of the study and included for analysis. The mean patient age was 42.7 years, $1030(50.6 \%)$ patients were male, and 1139 (56.0\%) had Crohn's disease (CD). Among the 2035 patients, 538 (26.4\%) experienced COVID19 hospitalization or death, and $64(3.1 \%)$ died. The most common comorbidity was hypertension (12.6\%), followed by cardiovascular disease (7.1\%) and diabetes $(5.8 \%)$. The study population is further summarized in Table 1.

In the first analysis, we performed separate logistic regressions for each comorbidity, adjusting for age, sex, and the other variables shown in Table 2. All examined comorbidities were statistically significant except for a history of stroke [adjusted odds ratio (aOR) 1.62, 95\% confidence interval (CI) 0.71-3.70] and obesity (aOR 1.07, 95\% CI 0.80-1.43). Most comorbidities were associated with a two to threefold odds of hospitalization or death (Table 2). The highest aORs were observed for CKD (aOR 3.73, 95\% CI 1.84-7.56) and COPD (aOR 3.55, 95\% CI 1.66-7.58).

Next, we evaluated the independent effects of each comorbidity in a single model, adjusting for all other comorbidities, as patients with one comorbidity could be more likely
Table 1 Baseline demographic and clinical characteristics of IBD patients with COVID-19 reported to the SECURE-IBD registry until August 2020

\begin{tabular}{|c|c|c|}
\hline \multirow[t]{2}{*}{ Characteristic $^{\mathrm{a}}$} & \multicolumn{2}{|c|}{ All patients } \\
\hline & $N$ & $\%$ \\
\hline Total number of patients & 2035 & \\
\hline Age (mean, SD) & 42.7 & 17.9 \\
\hline Male gender & 1030 & $51 \%$ \\
\hline \multicolumn{3}{|l|}{ Ethnicity } \\
\hline White & 1620 & $80 \%$ \\
\hline Black & 145 & $7 \%$ \\
\hline Asian & 112 & $6 \%$ \\
\hline Other & 156 & $7 \%$ \\
\hline \multicolumn{3}{|l|}{ Hispanic/Latinx } \\
\hline Yes & 352 & $17 \%$ \\
\hline No & 1281 & $63 \%$ \\
\hline Unknown & 402 & $20 \%$ \\
\hline \multicolumn{3}{|l|}{ Disease type } \\
\hline Crohn's disease & 1139 & $56 \%$ \\
\hline Ulcerative colitis & 854 & $42 \%$ \\
\hline IBD unspecified & 42 & $2 \%$ \\
\hline Biologic therapy & 1203 & $59 \%$ \\
\hline 5-ASA & 630 & $31 \%$ \\
\hline Immunomodulators & 460 & $23 \%$ \\
\hline Corticosteroids & 206 & $10 \%$ \\
\hline Janus kinase Inhibitors & 25 & $1 \%$ \\
\hline Other IBD treatment & 67 & $3 \%$ \\
\hline No IBD treatment & 65 & $3 \%$ \\
\hline Cardiovascular disease & 144 & $7 \%$ \\
\hline Diabetes & 118 & $6 \%$ \\
\hline Asthma & 107 & $5 \%$ \\
\hline COPD & 43 & $2 \%$ \\
\hline Other chronic lung disease & 34 & $2 \%$ \\
\hline Hypertension & 256 & $13 \%$ \\
\hline Cancer & 43 & $2 \%$ \\
\hline History of stroke & 29 & $1 \%$ \\
\hline Chronic kidney disease & 48 & $2 \%$ \\
\hline Chronic liver disease & 69 & $3 \%$ \\
\hline Obesity $(\mathrm{BMI} \geq 30)$ & 335 & $16 \%$ \\
\hline Cigarette smoker & 84 & $4 \%$ \\
\hline Other tobacco user & 16 & $1 \%$ \\
\hline
\end{tabular}

SECURE-IBD Surveillance Epidemiology of Coronavirus Under Research Exclusion for Inflammatory Bowel Disease, COVID-19 coronavirus disease 2019, SD standard deviation, 5-ASA 5-aminosalicylic acid, $I B D$ inflammatory bowel disease, $C O P D$ chronic obstructive pulmonary disease, $B M I$ body mass index

${ }^{a}$ Unless otherwise specified, percentages do not include missing values or "unknown." For all characteristics, less than $4 \%$ of data was missing and unknown, respectively, for each category. Percentages and $\mathrm{n}$ from each subcategory may not add up to the exact number of total reported cases due to missing values and/or non-mutually exclusive variables 
Table 2 Association of non-IBD comorbidities and adverse COVID19 outcomes (hospitalization and death) in IBD patients with COVID-19 in the SECURE-IBD registry in separate models

\begin{tabular}{llr}
\hline Comorbidity & aOR $(95 \% \mathrm{CI})^{\mathrm{a}}$ & $P$ value \\
\hline Asthma $^{\mathrm{b}}$ & $1.88(1.21-2.92)$ & 0.005 \\
Cancer $^{\mathrm{c}}$ & $1.96(1.01-3.81)$ & 0.047 \\
Cardiovascular disease $^{\mathrm{d}}$ & $1.86(1.25-2.78)$ & 0.002 \\
Chronic liver disease $^{\mathrm{e}}$ & $2.33(1.39-3.89)$ & 0.001 \\
Chronic kidney disease $^{\mathrm{f}}$ & $3.73(1.84-7.56)$ & $<0.001$ \\
COPD $^{\mathrm{g}}$ & $3.55(1.66-7.58)$ & 0.001 \\
Diabetes $^{\mathrm{h}}$ & $2.73(1.79-4.18)$ & $<0.001$ \\
History of stroke $^{\mathrm{i}}$ & $1.62(0.71-3.70)$ & 0.249 \\
Hypertension $^{\mathrm{j}}$ & $2.01(1.48-2.73)$ & $<0.001$ \\
Obesity $^{\mathrm{k}}$ & $1.07(0.80-1.43)$ & 0.635 \\
Other chronic lung disease $^{\mathrm{l}}$ & $3.02(1.39-6.55)$ & 0.005 \\
\hline
\end{tabular}

SECURE-IBD Surveillance Epidemiology of Coronavirus Under Research Exclusion for Inflammatory Bowel Disease, COVID-19 coronavirus disease 2019, COPD chronic obstructive pulmonary disease, $a O R$ adjusted odds ratio, $C I$ confidence interval

${ }^{a}$ Each comorbidity was tested in a separate model after adjusting for variables other than comorbidities that were significantly associated with the outcome on bivariate analyses

${ }^{\mathrm{b}}$ Adjusted for age, sex, race, and ethnicity

${ }^{c}$ Adjusted for age, sex, race, biologic therapy, 5-ASA, immunomodulators, and cigarette use

${ }^{\mathrm{d}}$ Adjusted for age, sex, biologic therapy, 5-ASA, corticosteroids, and cigarette use

${ }^{\mathrm{e}}$ Adjusted for age and sex

${ }^{\mathrm{f}}$ Adjusted for age, sex, race, biologic therapy, and corticosteroids

${ }^{g}$ Adjusted for age, sex, biologic therapy, 5-ASA, and corticosteroids

${ }^{\mathrm{h}}$ Adjusted for age, sex, race, ethnicity, biologic therapy, and 5-ASA

${ }^{\mathrm{i}}$ Adjusted for age, sex, race, biologic therapy, and cigarette use

${ }^{\mathrm{j}}$ Adjusted for age, sex, race, biologic therapy, 5-ASA, and cigarette use

${ }^{\mathrm{k}}$ Adjusted for age, sex, biologic therapy, 5-ASA, and corticosteroids

${ }^{1}$ Adjusted for age, sex, race, biologic therapy, 5-ASA, corticosteroids, and cigarette use

to have others (Table 3). As previously observed, increased patient age (aOR 1.03, 95\% CI 1.02-1.04) and use of corticosteroids (aOR 2.90, 95\% CI 2.09-4.03) were associated with higher odds of hospitalization or death. Associations with male sex (aOR 1.22, 95\% CI 0.97-1.53) and use of 5-ASA (aOR 1.13 , 95\% CI 0.88-1.45) were not statistically significant. The use of biologic therapy was observed to have a protective association (aOR $0.51,95 \%$ CI $0.40-0.65$ ). Overall, the effect size for most comorbidities was similar in this fully adjusted analysis compared to individual analysis of each comorbidity. CKD (aOR 3.02, 95\% CI 1.45-6.31) and COPD (aOR 2.92, 95\% CI 1.32-6.48) remained the most detrimental comorbidities. However, the odds of association of adverse COVID-19 outcomes with cancer (aOR 1.87, 95\% CI 0.94-3.75) and cardiovascular disease (aOR 1.34, 95\%
Table 3 Association of non-IBD comorbidities and adverse COVID19 outcomes (hospitalization and death) in IBD patients with COVID-19 in the SECURE-IBD registry in a composite model

\begin{tabular}{llr}
\hline Variable $^{\mathrm{a}}$ & $\mathrm{aOR}(95 \% \mathrm{CI})$ & $P$ value \\
\hline Asthma & $1.96(1.24-3.11)$ & 0.004 \\
Diabetes & $1.95(1.22-3.11)$ & 0.005 \\
Cancer & $1.87(0.94-3.75)$ & 0.076 \\
Cardiovascular disease & $1.34(0.87-2.06)$ & 0.186 \\
Chronic liver disease & $1.97(1.14-3.39)$ & 0.015 \\
Chronic kidney disease & $3.02(1.45-6.31)$ & 0.003 \\
COPD & $2.92(1.32-6.48)$ & 0.008 \\
Hypertension & $1.50(1.07-2.10)$ & 0.018 \\
Other chronic lung disease & $2.38(1.06-5.35)$ & 0.035 \\
Age & $1.03(1.02-1.04)$ & $<0.001$ \\
Male sex & $1.22(0.97-1.53)$ & 0.083 \\
5-ASA & $1.13(0.88-1.45)$ & 0.343 \\
Corticosteroids & $2.90(2.09-4.03)$ & $<0.001$ \\
Biologic therapy & $0.51(0.40-0.65)$ & $<0.001$ \\
\hline
\end{tabular}

SECURE-IBD Surveillance Epidemiology of Coronavirus Under Research Exclusion for Inflammatory Bowel Disease, COVID-19 coronavirus disease 2019, COPD chronic obstructive pulmonary disease, 5-ASA 5-aminosalicylic acid, $a O R$ adjusted odds ratio, $C I$ confidence interval

${ }^{\text {a }}$ These variables were incorporated into a multivariable logistic regression, with hospitalization or death as the response variable

CI 0.87-2.06) were attenuated and no longer statistically significant in the fully adjusted model. Directions and magnitudes of all effects were similar when the outcome variable was a need for mechanical ventilation or patient death.

\section{Discussion}

We used an international dataset of over 2000 reports of COVID-19 infection in patients with IBD to understand the relative effects of comorbidities on the severity of COVID19. Consistent with literature in the general population, we observed that most non-IBD comorbidities are associated with a two to threefold risk of hospitalization or death. Given the high comorbidity burden for patients with IBD, these findings represent an important factor in risk-stratifying patients during the course of this pandemic and may be used to inform clinical care discussions as well as lifestyle decisions regarding returning to work, school, and other normal activities.

This study builds upon prior work indicating that, among patients with IBD, the presence of comorbidities is a risk factor for more severe COVID-19. Bezzio et al. found a strong association between Charlson Comorbidity Index and death among patients with IBD affected by COVID-19 (OR 16.66, 95\% CI 1.80-153.9), research by Singh et al. 
indicated that patients with IBD that experienced severe COVID-19 had a greater presence of comorbidities $(P<0.05$ for essential hypertension, diabetes mellitus, ischemic heart diseases, heart failure, cerebrovascular diseases, and chronic kidney disease), and prior work from our group indicated that the presence of at least two non-IBD comorbidities was positively associated with the risk of intensive care hospitalization and/or death (aOR 2.9, 95\% CI 1.1-7.8) [8, 18, $21]$. Our analyses similarly demonstrated that most comorbidities were associated with worse COVID-19 outcomes. Furthermore, each statistically significant comorbidity had an odds ratio greater than 1.8 , indicating that the resulting increase in the odds of hospitalization or death is relatively high. CKD and COPD conferred the greatest risk, both with odds ratios greater than 3.5. After a second analysis adjusting for the independent effects of each comorbidity, CKD and COPD remained the most detrimental comorbidities. The associations of these two comorbidities with severity of COVID-19 infection are consistent with prior research. Studies conducted within the general population that examined the effects of both CKD and COPD showed both comorbidities to confer greatly increased risk of severe infection. Thus, our findings in the IBD population are consistent with this prior literature [22, 23]. A history of stroke was not statistically significant. This result is surprising, as prior research suggested that this comorbidity is a significant predictor of severe COVID-19 infection among the general population [24]. This may be attributable to the fact that stroke patients are often placed on oral anticoagulants, which may have a protective effect in the prothrombotic milieu of COVID-19 infection [25]. Furthermore, obesity was not statistically significant. This, too, is in contrast to other research suggesting that obesity is a strongly detrimental factor in COVID-19 infection in the general population [26]. However, this prior study did not account for the same comorbidities as did this research, and it is possible that the observed effect of obesity was mediated by other comorbid conditions.

As with prior analyses of data from SECURE-IBD, we found that steroid use was associated with adverse COVID19 outcomes while biologic use was protective [18]. In contrast to prior work, associations between 5-ASA/mesalamine and COVID-19 hospitalization or death were not significant, perhaps owing to tighter control of confounding by nonIBD comorbidities in this larger analysis with more granular assessment of individual comorbidities.

This study's strength stems largely from the SECUREIBD database, a large international registry created through the collaborative effort of healthcare providers across the world which allowed us to analyze over 2,000 COVID-19 infections in patients with IBD. Nevertheless, IBD providers may not have fully reported the presence of comorbidities, leading to misclassification of exposure that may have slightly attenuated observed associations. Another limitation is the potential for reporting bias. Data came from a convenience sample, which seems to over-represent white patients from the United States. Furthermore, the dataset might also over-represent severe infection. It may be that a doctor is more likely to be aware of or report a COVID-19 infection that presents more severe symptoms than one that does not. Further, it was unexpected that cancer was not significantly linked with severe infection in the independent effects analysis, as it has previously shown association with severe infection in the general population [7]. Perhaps this discrepancy was due to the lack of granularity with regards to cancer type, stage, treatment, and other clinical details in the SECURE IBD database which may have diluted the effect of active cancer, including metastatic cancer or cancers currently treated with chemotherapy. Lastly, it is important to acknowledge that this analysis is observational, and the correlation described throughout this research does not necessarily imply causation due to the possibility of unmeasured confounding and/or pure chance.

As COVID-19 continues to sweep through our society as we approach a new normal, it is important for patients and doctors to be able to stratify and assess risk, especially for patients with IBD who may already be predisposed to worse clinical outcomes. As expected, we found patient age and corticosteroid use linked with more severe COVID-19, as were many of the comorbidities we analyzed. In particular, CKD and COPD greatly increased risk of severe infection. These findings can be used to help patients and their providers make informed decisions about returning to normal activities and the level of precautions that should be taken.

\section{Conclusion}

Many comorbidities are associated with severe COVID19 infection among patients with IBD. CKD and COPD were the most detrimental of all comorbidities in the study, both conferring approximately a threefold-increased risk of severe infection.

Funding This work was funded by the Helmsley Charitable Trust (2003-04445), National Center for Advancing Translational Sciences (UL1TR002489), a T32DK007634 (EJB), and a K23KD11199501A1 (RCU). Additional funding provided by Pfizer, Takeda, Janssen, Abbvie, Eli Lilly, Genentech, Boehringer Ingelheim, Bristol Myers Squibb, Celtrion, and Arenapharm.

\section{Declarations}

Conflict of interest RP reports no conflict of interest. XZ reports no conflict of interest. RCU has served as a consultant and/or advisory board member for Bristol Myers Squibb, Eli Lilly, Janssen, Pfizer and Takeda. He has received research support from AbbVie, Boehringer Ingelheim and Pfizer. He is supported by a Career Development 
Award from the National Institutes of Health (K23KD111995-01A1). EJB reports no conflict of interest. MA reports no conflict of interest. JFC reports receiving research grants from AbbVie, Janssen Pharmaceuticals and Takeda; receiving payment for lectures from AbbVie, Amgen, Allergan, Inc. Ferring Pharmaceuticals, Shire, and Takeda; receiving consulting fees from AbbVie, Amgen, Arena Pharmaceuticals, Boehringer Ingelheim, Celgene Corporation, Celltrion, Eli Lilly, Enterome, Ferring Pharmaceuticals, Genentech, Janssen Pharmaceuticals, Landos, Ipsen, Medimmune, Merck, Novartis, Pfizer, Shire, Takeda, Tigenix, Viela bio; and hold stock options in Intestinal Biotech Development and Genfit. MDK has consulted for Abbvie, Janssen, Pfizer, and Takeda, is a shareholder in Johnson \& Johnson, and has received research support from Pfizer, Takeda, Janssen, Abbvie, Lilly, Genentech, Boehringer Ingelheim, Bristol Myers Squibb, Celtrion, and Arenapharm.

Ethical statement Each SECURE-IBD survey item met criteria for de-identified data, in accordance with the HIPAA Safe Harbor De-Identification standards. The UNC-Chapel Hill Office for Human Research Ethics has determined that the storage and analysis of de-identified data for this project does not constitute human subjects research as defined under federal regulations [45 CFR 46.102 and 21 CFR 56.102] and does not require IRB approval.

Open Access This article is licensed under a Creative Commons Attribution-NonCommercial 4.0 International License, which permits any non-commercial use, sharing, adaptation, distribution and reproduction in any medium or format, as long as you give appropriate credit to the original author(s) and the source, provide a link to the Creative Commons licence, and indicate if changes were made. The images or other third party material in this article are included in the article's Creative Commons licence, unless indicated otherwise in a credit line to the material. If material is not included in the article's Creative Commons licence and your intended use is not permitted by statutory regulation or exceeds the permitted use, you will need to obtain permission directly from the copyright holder. To view a copy of this licence, visit http://creativecommons.org/licenses/by-nc/4.0/.

\section{References}

1. Velavan TP, Meyer CG. The COVID-19 epidemic. Trop Med Int Health 2020;25:278-280. https://doi.org/10.1111/tmi.13383.

2. Cao X. COVID-19: immunopathology and its implications for therapy. Nat Rev Immunol 2020;20:269-270. https://doi.org/10. 1038/s41577-020-0308-3.

3. Allen J, Almukhtar S, Aufrichtig A, Barnard A, Bloch M, Cahalan S, Cai W, Calderone J, Collins K, Conlen M, Cook L, Gianordoli G, Harmon A, Harris R, Hassan A, Huang J, Issawi D, Ivory D, Lai K, Lemonides A, Lutz E, McCann A, Oppel R, Patel J, Saldanha A, Semple K, Seroussi A, Shaver J, Singhvi A, Smart C, Smith M, Sun A, Taylor R, Watkins D, Williams T, Wu J, Yourishl K. Coronavirus World Map: Tracking the Global Outbreak. The New York Times, 22 Mar. 2021. www.nytimes.com/interactive/ 2020/world/coronavirus-maps.html.

4. Li H, Wang S, Zhong F, Bao W, Li Y, Liu L, Wang H, He Y. Agedependent risks of incidence and mortality of COVID-19 in Hubei Province and other parts of China. Front Med. 2020. https://doi. org/10.3389/fmed.2020.00190.

5. Baud D, Qi X, Nielsen-Saines K, Musso D, Pomar L, Favre G. Real estimates of mortality following COVID-19 infection. Lancet Infect Dis 2020;20:773. https://doi.org/10.1016/s1473-3099(20) 30195-x.
6. Wiley Z, Kubes J, Cobb J, Jacob J, Franks N, Plantinga L, Lea J. Age, comorbid conditions, and racial disparities in COVID-19 outcomes. J Racial Ethn Health Disparities. 2021. https://doi.org/ 10.1007/s40615-020-00934-0.

7. Guan W, Liang W, Zhao Y, Liang H, Chen Z, Li Y, Liu X, Chen R, Tang C, Wang T, Ou C, Li L, Chen P, Sang L, Wang W, Li J, Li C, Ou L, Cheng B, Xiong S, Ni Z, Xiang J, Hu Y, Liu L, Shan H, Lei C, Peng Y, Wei L, Liu Y,Hu Y, Peng P, Wang J, Liu J, Chen Z, Li G, Zheng Z, Qiu S, Luo J, Ye C, Zhu S, Cheng L, Ye F, Li S, Zheng J, Zhang N, Zhong N, He J. Comorbidity and Its Impact on 1590 Patients with COVID-19 in China: A Nationwide Analysis. European Respiratory Society, 1 May 2020. https://erj.ersjournals. com/content/55/5/2000547.short.

8. Bezzio C, Saibeni S, Variola A, Allocca M, Massari A, Garardi V, Casini V, Ricci C, Zingone F, Amato A, Caprioli F, Lenti M, Viganò C, Ascolani M, Bossa F, Castiglione F, Cortelezzi C, Grossi L, Milla M, Morganti D, Pastorelli L, Ribaldone D, Sartini A, Soriano A, Manes G, Danese S, Fantini M, Armuzzi A, Daperno M, Fiorino G. Outcomes of COVID-19 in 79 patients with IBD in Italy: an IG-IBD study. Gut 2020;69:1213-1217. https://doi.org/10.1136/gutjnl-2020-321411.

9. D’Amico F, Danese S, Peyrin-Biroulet L. Systematic review on inflammatory bowel disease patients with coronavirus disease 2019: it is time to take stock. Clin Gastroenterol Hepatol 2020;18:2689-2700. https://doi.org/10.1016/j.cgh.2020.08.003.

10. Pepys M, Druguet M, Klass H, Dash A, Mirjah D, Petrie A. Immunological studies in inflammatory bowel disease. In Ciba Foundation Symposium 46-Immunology of the Gut Novartis Foundation Symposia, 2008, pp. 283-304. https://doi.org/10. 1002/9780470720288.ch14.

11. Biancone L, Scopinaro F, Maletta $\mathrm{M}$ et al. Circulating D dimer in inflammatory bowel disease. Ital J Gastroenterol 1994;26:116-120.

12. Gupta S. Elevated Level of C Reactive Protein May Predict Risk for Worsening COVID-19. Infectious Disease Advisor, 14 May 2020. www.infectiousdiseaseadvisor.com/home/topics/covid 19/high-crp-associated-with-increased-likelihood-of-progr ession-to-severe-covid-19/.

13. Farkas J. PulmCrit Wee: D-Dimer Cutoffs to Predict Thrombosis in COVID-19. EMCrit Project, 10 April 2020. http://emcrit.org/ pulmcrit/dimer-cutoff-covid/.

14. Ananthakrishnan AN, Mcginley EL. Infection-related hospitalizations are associated with increased mortality in patients with inflammatory bowel diseases. J Crohn's Colitis 2013;7:107-112. https://doi.org/10.1016/j.crohns.2012.02.015.

15. Mehta P, McAuley D, Brown M, Sanchez E, Tattersall R, Manson J. COVID-19: consider cytokine storm syndromes and immunosuppression. Lancet 2020;395:1033-1034. https://doi.org/10. 1016/s0140-6736(20)30628-0.

16. Horby P, Lim W, Emberson J, Haynes R, Landray M. Effect of dexamethasone in hospitalized patients with COVID-19: preliminary report. MedRxiv. 2020. https://doi.org/10.1101/2020.06.22. 20137273.

17. Haapamäki J, Roine R, Turunen U, Färkkilä M, Arkkilla P. Increased risk for coronary heart disease, asthma, and connective tissue diseases in inflammatory bowel disease. J Crohn's Colitis 2011;5:41-47. https://doi.org/10.1016/j.crohns.2010.09.008.

18. Brenner E, Ungaro R, Gearry R, Kaplan G, Kissous-Hunt M, Lewis J, Ng S, Rahier J, Reinisch W, Ruemmele F, Steinwurz F, Underwood F, Zhang X, Colombel J, Kappelman M. CME exam 1: corticosteroids, but not TNF antagonists, are associated with adverse COVID-19 outcomes in patients with inflammatory bowel diseases: results from an international registry. Gastroenterology. 2020. https://doi.org/10.1053/j.gastro.2020.07.020. 
19. R Core Team. R: A Language and Environment for Statistical Computing. Vienna: R Foundation for Statistical Computing; 2019. https://www.R-project.org/.

20. Wickham H, Averick M, Bryan J, Chang W, McGowan L, François R, Grolemund G, Hayes A, Henry L, Hester J, Kuhn M, Pedersen T, Miller E, Bache S, Müller K, Ooms J, Robinson D, Seidel D, Spinu V, Takahashi K, Vaughan D, Wilke C, Woo K, Yutani H. Welcome to the tidyverse. J Open Source Softw 2019;4:1686. https://doi.org/10.21105/joss.01686.

21. Singh S, Khan A, Chowdhry M, Bilal M, Kochhar G, Clarke K. Risk of severe coronavirus disease 2019 in patients with inflammatory bowel disease in the United States: a multicenter research network study. Gastroenterology. 2020. https://doi.org/10.1053/j. gastro.2020.06.003.

22. Henry BM, Lippi G. Chronic kidney disease is associated with severe coronavirus disease 2019 (COVID-19) infection. Int Urol Nephrol 2020;52:1193-1194. https://doi.org/10.1007/ s11255-020-02451-9.

23. Zhao Q, Meng M, Kumar R, Wu Y, Huang J, Lian N, Deng Y, Lin S. The impact of COPD and smoking history on the severity of COVID-19: a systemic review and meta-analysis. J Med Virol. 2020. https://doi.org/10.1002/jmv.25889.
24. Qin C, Zhou L, Hu Z, Yang S, Zhang S, Chen M, Yu H, Tian D, Wang W. Clinical characteristics and outcomes of COVID19 patients with a history of stroke in Wuhan, China. Stroke 2020;51:2219-2223. https://doi.org/10.1161/strokeaha.120. 030365 .

25. Rico-Mesa J, Rosas D, Ahmadian-Tehrani A, White A, Anderson A, Chilton R. The role of anticoagulation in COVID-19-induced hypercoagulability. Curr Cardiol Rep. 2020. https://doi.org/10. 1007/s11886-020-01328-8.

26. Cai Q, Chen F, Wang T, Luo F, Liu X, Wu Q, He Q, Wang Z, Liu Y, Liu L, Chen J, Xu L. Obesity and COVID-19 severity in a designated hospital in Shenzhen, China. Diabetes Care 2020;43:1392-1398. https://doi.org/10.2337/dc20-0576.

Publisher's Note Springer Nature remains neutral with regard to jurisdictional claims in published maps and institutional affiliations. 